\title{
Computer Assisted Optimization of Prosthetic Socket Design for the Lower Limb Amputees Using 3-D Scan
}

\author{
Fee von WALDENFELS ${ }^{* 1}$, Stefan RAITH ${ }^{1}$, Maximilian EDER ${ }^{1}$, Alexander VOLF ${ }^{1}$, Jalil JALALI ${ }^{2}$, \\ Laszlo KOVACS ${ }^{1}$
}

${ }^{1}$ Research Group CAPS (Computer Aided Plastic Surgery) - Klinik und Poliklinik für Plastische Chirurgie
und Handchirurgie, Klinikum rechts der Isar, Technische Universität München, Germany
${ }^{2}$ Institute of Medical Engineering at the Technische Universität München (IMETUM), Garching, Germany

\begin{abstract}
Introduction:

Customary prosthetic socket construction and fabrication process do not take patient specific parameters into account, instead they are based on subjective estimations, competence and capabilities of the orthopedic technician. Therefore a high rate of inappropriate prosthetic supplies is caused to the disadvantage of the amputation patient who suffers from wounds due to excessive pressure. A development of objective planning systems is required, in order to gain higher quality in the prosthetic socket construction. The central aim of the presented work was to improve the currently empirical process of prosthesis design in orthopedic technology with the aid of 3-D scan of the amputation stump, modern imaging techniques (MRI) and computer technology (FEA).
\end{abstract}

\section{Method:}

Regarding a 3-D scan protocol the amputation stump of 10 patients were scanned in upright position using a 3-D linear laser scanner. Furthermore magnet resonance imaging (MRI) was performed in lying supine position, which produced non-negligible deformation in the soft tissue of the stump. MRI datasets were segmented into different compartments representing fat, muscle and bone using a special software workflow. The segmented geometries were fitted in the surface geometry of the upright 3-D scan. Therefore a simplified FE model was built in consideration of stiffness of soft tissue and bone. The calculated models reproduced the inner anatomical structure of the stump. The described method has been validated on a patient where both upright and regular supine MR image data were accessible in addition to 3-D surface scans with the aid of software calculating the 3-D compare (mean standard deviation in $\mathrm{mm}$ ) of the 3-D models. The results of the deformation calculations of the patients were validated in FEA simulations and gait analysis.

\section{Results:}

Using 3-D compare of the obvious deformed MRI model in lying position in comparison to the upright MRI model the mean standard deviation (SD) was $13.5 \mathrm{~mm}$. The 3-D comparison of the reversely calculated MRI model in comparison to the upright MRI model showed a mean standard deviation of $5.8 \mathrm{~mm}$ (improvement factor 2.34). The mean standard deviation of $5.8 \mathrm{~mm}$ did not exceed the mean edge length of the finite elements, thus the results are sufficiently accurate for the following simulations performed by FEA software.

\section{Conclusion:}

Validation results show a considerable improvement from the deformed MRI model in comparison to the reversely calculated MRI model, which is important for a realistic and physically based computer assisted design of the prosthetic socket. Based on patient-specific 3-D model, 3-D visualization, quantification and simulation of the individual biomechanical tissue changes in the amputation stump during the interaction with the prosthetic socket can be evaluated using finite element analysis (FEA).

Keywords: 3-D scan, MRI, finite element analysis, prosthetic socket, optimization

*fee.waldenfels@caps.me.tum.de; +49 89 28910857; www.caps.me.tum.de 


\section{Introduction}

\subsection{Customary prosthetic socket construction}

The incidence of amputations in Germany is - in comparison to other European countries - relatively high, with more than 60,000 amputations per year [1]. Experts predict an increase of the number of amputations in the future despite improved medical prevention and successful treatment of potential risk factors. This fact will be reflected in the future according to demographic changes related to the negative correlation between increased health care system costs and decreased social insurance proceeds $[2,3]$. The most important medical therapy concept for amputees is to provide prosthetic supply and to enable this patient collective to participate in active life and to socially reintegrate [4]. Due to this therapy, patients regain mobility, can still feed themselves and even partially reintegrate to professional life, whereby, potential occurring care-giving expenses can be reduced. However, the prosthetic socket must not create too high pressure to the stump's soft tissue in order to avoid low perfusion areas, pressure marks and wound healing problems (Fig. 1.). A homogeneous pressure distribution over the entire stump must be obtained under static and dynamic pressure conditions. In addition, an optimally shaped prosthetic socket must consider the individual soft tissue properties of the amputation stump. These facts pose an enormous patient specific challenge to the orthopedic technician [5].



Fig. 1. High pressure to the stump's soft tissue can cause low perfusion areas, pressure marks and wound healing problems.

\subsection{Aim of the presented research work}

The improvement of the currently empirical process of prosthesis design in orthopedic technology with the aid of modern imaging techniques computer technology by taking into account patient-specific consistence of the amputation stump is the central aim of the work. The cooperation project (COPKOComputer Aided Optimization of Prosthetic Socket Construction in Orthopedics) is peer-reviewed and funded by the Bavarian Research Foundation (www.forschungsstiftung.de) in Germany. To capture and simulate the complex processes of changing soft tissue properties during the prosthetic treatment, especially in the interaction with the prosthetic socket, the development of a realistic, anatomical 3-D model of the amputation stump is required. Using modern gait laboratory analysis and pressure distribution measurements, the biomechanical tissue changes can be quantified and are considered for the creation of a physically realistic virtual 3-D model of the amputation stump. Based on patient-specific 3-D models, the 3-D visualization, quantification and simulation of the individual biomechanical tissue changes in the amputation stump during the interaction with the prosthetic socket was evaluated using finite element analysis. The computer-aided visualization and virtual simulation of soft tissue deformation and of the contact forces in the interface between the patientspecific deformable model and the amputation stump at the computer individually planned model of the prosthetic socket will serve as basis for a reproducible construction and fabrication of individually adjusted exoskeleton prosthetic socket systems as well as a computer-simulated "virtual try-on" before construction and fabrication of the prosthesis even in the absence of the patients. 


\section{Method}

\subsection{3-D surface scanning}

3-D surface imaging systems enables a more precise capturing and quantification of the external shape of the amputation stump [6]. 3-D surface scanning was performed by a surface scanner using laser triangulation (Konica Minolta Co., Ltd., Osaka, Japan). Regarding a 3-D scan protocol realigned to preliminary studies examining breast shape measurements $[7,8,9,10,11]$ - the amputation stump of the patient were scanned in upright position under standardized lighting conditions (light intensity 350-4000 lux). The amputation stump was hanging down without muscle tension during imaging. The 3-D Scan was taken from multiple directions (a-p: +45 degrees, $0,-45$ degrees; $p$-a: +45 degrees, 0, - 45 degrees), to receive an image of the entire amputation stump (Fig. 2).
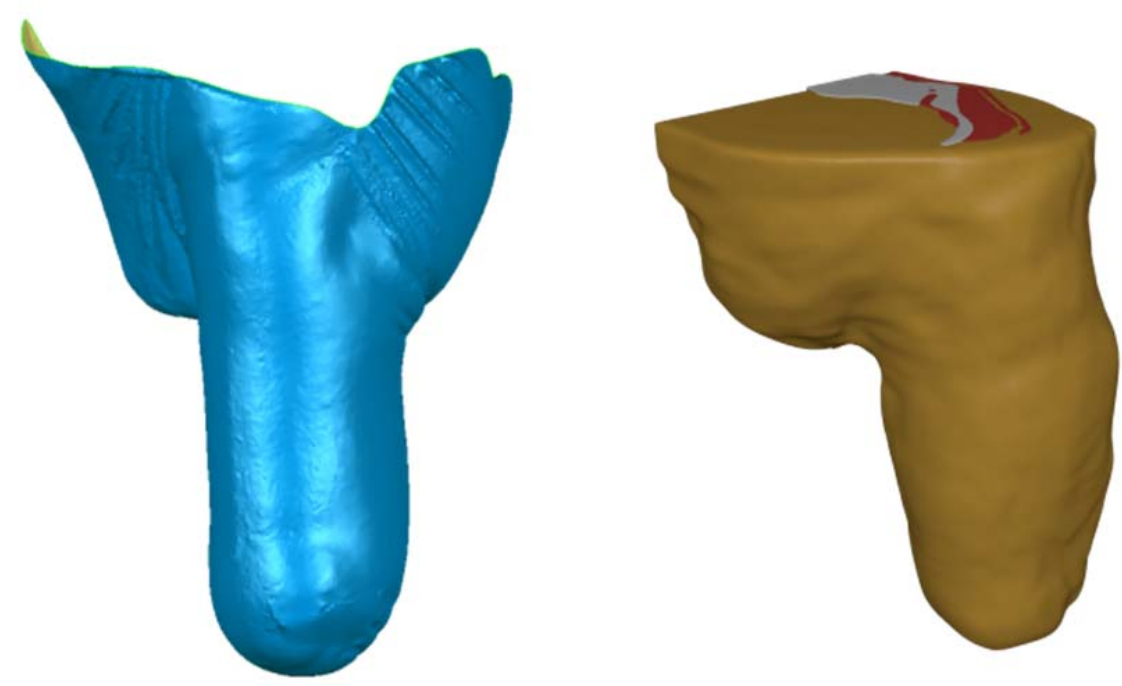

Fig. 2. 3-D surface scan of an amputation stump (I) and corresponding MRI data (r).

The recalculation of the amputation stump, deformed by lying in supine position in the MRI, back in the upright position provides the source for the development of a realistic, anatomical 3-D model for the described project and exemplifies an innovative method. The described process has been validated on a patient where both upright and regular supine MR image data were accessible in addition to 3-D surface scans with the aid of a software (Raindrop Geomagic Studio 10®, Raindrop Geomagic, Inc., NC, USA) calculating the 3-D compare (mean standard deviation in $\mathrm{mm}$ ) of the 3-D models.

\subsection{Magnet resonance imaging (MRI)}

Patients participating in the project underwent MRI, after being informed of the investigation and having signed a patient consent, in a Philips Achieva 1,5 Tesla MRI Scanner (Philips Medical Systems DMC GmbH, Hamburg, Germany) using T1-weighted imaging sequence. For this purpose, an MRI protocol to ensure a standardized examination was developed. The stump of the lying patient in supine position is examined without prosthesis from the distal end of the stump to the pelvic height (anterior superior iliac spine). The amputation stump is supported by two pads (medially and laterally) in order to site the stump in the right position for the MRI performance without producing deformation to the soft tissue. The resultant T1-weighted images are transferred in DICOM files into the program Mimics ${ }^{\circledR}$ (Materialise, Leuven, Belgium) and were segmented into different compartments representing fat, muscle and bone (Fig. 3.). 


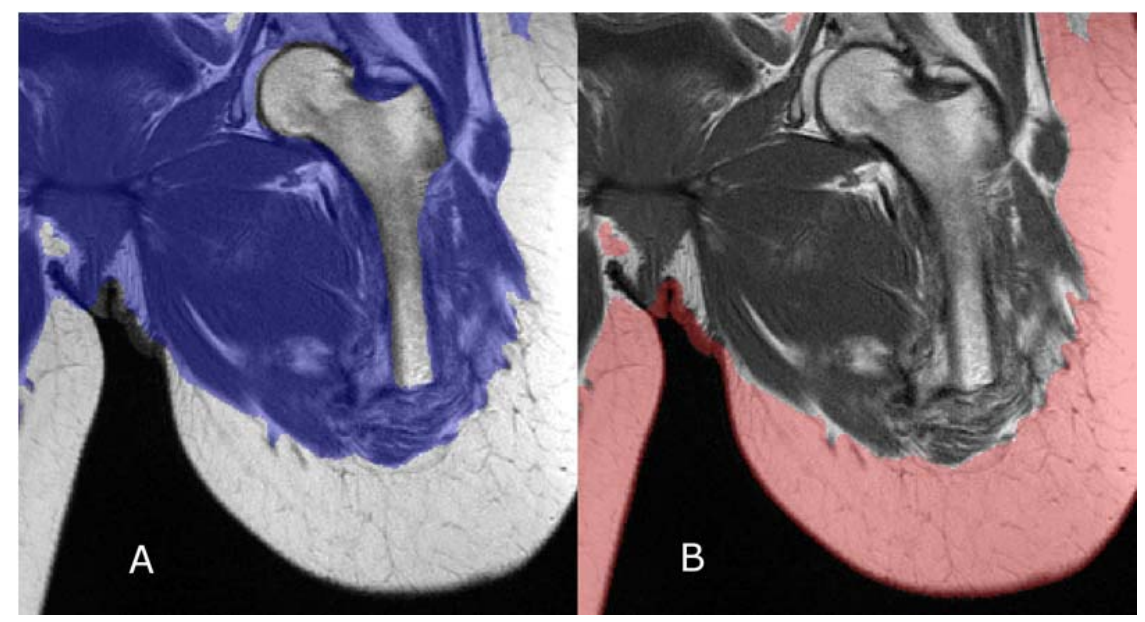

Fig. 3. Different segmented compartments: A) muscle tissue B) fat tissue.

The amputation stump lying on the scanner pad causes deformation in the dorsal soft tissue parts.

For the process of recalculation and to validate the constructed anatomical volume models, an upright MRI scan of one patient was performed in standing position with and without an optimal fitted prosthesis socket and in lying position with and without an optimal fitted prosthesis socket.

\subsection{Finite element analysis (FEA)}

The physical behavior of biological tissue can be virtually simulated using specific numerical formulas. With FEM it is possible to quantify the deformation of anatomical structures caused by the prosthetic socket compression. The FEM is well established for industrial purposes and is also applicable to medical and biomechanical objectives $[19,20]$. The segmented geometries were fitted in the surface geometry of the upright 3-D scan. Therefore a simplified FE model was built in consideration of stiffness of soft tissue and bone using a FEA-Software (ANSYS, CADFEM GmbH, Grafing, Germany). The calculated models reproduced the inner anatomical structure of the stump. The results of the deformation calculations of the patients were validated in FEA simulations and gait- and pressure analysis (Fig. 4.).
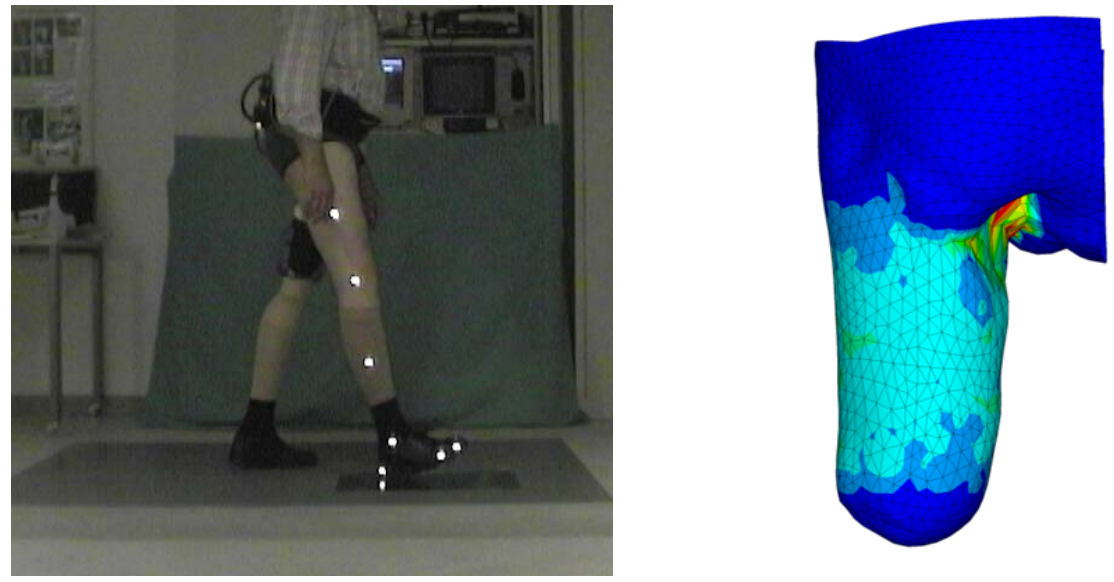

Fig. 4. Validation of the deformation calculations via gait analysis (left) and finite element analysis (right).

\subsection{Gait and pressure distribution analysis}

The described methods only represent static description of the amputation stump. Therefore, first approaches using gait and pressure distribution analysis are described to analyze the interaction between the stump and the socket and to evaluate the impact to the whole body movement of the patient [12-18]. These dynamic analyzing methods guarantee an objective quantification of the resulting interaction during the gait cycle and offer a visualization of the gait pattern (Fig. 4). 


\section{Results}

Using 3-D compare (Raindrop Geomagic Studio 10®, Raindrop Geomagic, Inc., NC, USA) of the obvious deformed MRI model in lying supine position in comparison to the upright MRI model the mean standard deviation (SD) was $13.5 \mathrm{~mm}$. The 3-D comparison of the reversely calculated MRI model in comparison to the upright MRI model showed a mean standard deviation of $5.8 \mathrm{~mm}$ (improvement factor 2.34) (Fig. 5. , Table 1). The mean standard deviation of $5.8 \mathrm{~mm}$ did not exceed the mean edge length of the finite elements, thus the results are sufficiently accurate for the following simulations performed by FEA software.

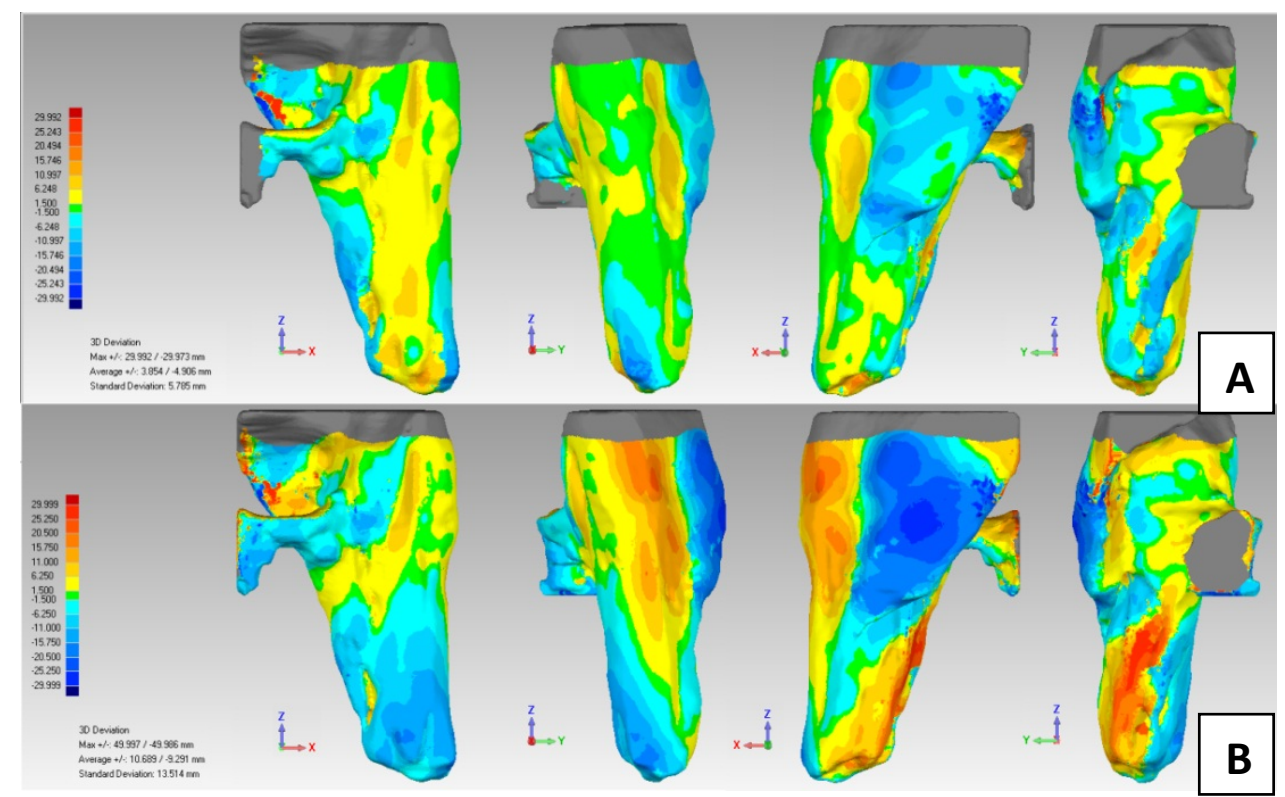

Fig. 5. 3-D compare: $(A)$ reversely calculated 3-D model in comparison to upright-MRI model and (B) lying supine $M R I$ model in comparison to upright MRI model.

\begin{tabular}{|c|c|c|c|}
\hline & calculated $\longleftrightarrow$ upright & supine $\longleftrightarrow$ upright & Improvement factor \\
\hline $\begin{array}{c}\text { mean standard } \\
\text { deviation in } \mathrm{mm}\end{array}$ & 5,785 & 13,514 & 2,34 \\
\hline
\end{tabular}

Table 1. Validation results of the 3-D compare.

Finite element analysis and data of the correspondent gait- and pressure analysis validate the application of the described method. The results of the final simulation geometry, received via the performed recalculation process, compared with the segmented 3-D model, serve as a validation tool for the applied numerical simulation model

\section{Conclusions}

The results described here, show that 3-D surface scanning is essential for the development of a realistic, anatomical 3-D volume model of the amputation stump. Validation results show a considerable improvement from the deformed MRI model in comparison to the reversely calculated MRI model, which is important for a realistic and physically based computer assisted design of the prosthetic socket. Based on patient-specific 3-D model and 3-D visualization quantification and simulation of the individual biomechanical tissue changes in the amputation stump during the interaction with the prosthetic socket can be evaluated using finite element analysis (FEA). 


\section{References}

1. Lawall, H. (2008) „Diabetes und Fußerkrankungen“ Deutscher Gesundheitsbericht, Diabetes 2008, Die Bestandsaufnahme. Vorgelegt von der Deutschen Diabetes-Union und dem Nationalen Aktionsforum Diabetes mellitus (NAFDM) zum Weltdiabetestag im November 2007

2. Heller G, Günster C, Swart E. Über die Häufigkeit von Amputationen unterer Extremitäten in Deutschland. Deutsche Medizinische Wochenschrift 2005; 130:1689-1690

3. Breuninger J. Beinprothesen für Oberschenkelamputierte, Diplom thesis, HfG Schwäbisch Gmünd, 2007

4. Roberts TL, Pasquina PF, Nelson VS, Flood KM, Bryant PR, Huang ME. Limb deficiency and prosthetic management. 4. Comorbidities associated with limb loss. Arch Phys Med Rehabil 2006; 87(Suppl. 1):S21-27

5. Kovacs et al., Patient-Specific Optimization of Prosthetic Socket Construction and Fabrication Using Innovative Manufacturing Processes: A Project in Progress, -"Mimics Innovation Award 2010"- Category 1: Innovative implant design system and the development of innovative medical procedures, Proceedings of the Medical Innovations Conference, 2010, Materialise Leuven, Belgium

6. Esquenazi A, Meier RH 3rd. Rehabilitation in limb deficiency. 4. Limb amputation. Arch Phys Med Rehabil. 1996 Mar;77(3 Suppl):S18-28. Review.

7. M. Eder, F. Waldenfels, A. Swobodnik, M. Klöppel, A. Pape, T. Schuster, S. Raith, E. Kitzler, N. Papadopulos, H. Machens und L. Kovacs, „Objective breast symmetry evaluation using 3-D surface imaging," Breast, p. [Epub ahead of print], 2011.

8. M. Eder, A. Schneider, H. Feussner, A. Zimmermann, C. Hoehnke, N. Papadopulos und E. Biemer, "Breast volume assessment based on 3D surface geometry: verification of the method using MR imaging," Biomed Tech, Bd. 53, pp. 112-121, 2008.

9. L. Kovacs, M. Eder, R. Hollweck, A. Zimmermann, M. Settles, A. Schneider, M. Endlich, A. Mueller, K. Schwenzer-Zimmerer, N. Papadopulos und E. Biemer, "Comparison between breast volume measurement using 3D surface imaging and classical techniques," The Breast, Bd. 16, pp. 137-145, 2007.

10. L. Kovacs, M. Eder, R. Hollweck, A. Zimmermann, M. Settles, A. Schneider, K. Udosic, K. Schwenzer-Zimmerer, N. Papadopulos und E. Biemer, „New Aspects of Breast Volume Measurement Using 3-Dimensional Surface Imaging," Ann Plast Surg, Bd. 57, pp. 602-610, 2006.

11. L. Kovacs, A. Yassouridis, A. Zimmermann, G. Brockmann, A. Woehnl, M. Blaschke, M. Eder, K. Schwenzer-Zimmerer, R. Rosenberg, N. Papadopulos und E. Biemer, "Optimization of 3dimensional imaging of the breast region with 3-dimensional laser scanners," Ann Plast Surg, Bd. 56, pp. 229-236, 2006.

12. Reynolds DP, Lord M. Interface load analysis for computer-aided design of below-knee prosthetic sockets. Med Biol Eng Comput 1992; 30:419-426

13. Rolock J, Tucker K. Development, evaluation, and use of a computer aided manufacturing technique based on "rapid prototyping" principles. Capabilities 1998; 7:1-2, 8-10

14. Yarnitzky G, Yizhar Z, Gefen A. Real-time subject-specific monitoring of internal deformations and stresses in the soft tissues of the foot: a new approach in gait analysis. J Biomech 2006; 39:26732689

15. Polliack AA, Craig DD, Sieh RC, Landsberger S, Mcneal DR. Laboratory and clinical tests of a prototype pressure sensor for clinical assessment of prosthetic socket fit. Prosthet Orthot Int 2002; 26:23-34

16. Goh JC, Lee PV, Chong SY. Stump/socket pressure profiles of the pressure cast prosthetic socket. Clin Biomech 2003; 18:237-243

17. Martinez-Villalpando EC, Herr H, Farrell M. Estimation of ground reaction force and zero moment point on a powered ankle-foot prosthesis. Conf Proc IEEE Eng Med Biol Soc 2007; 1:4687-1492

18. Goujon H, Bonnet X, Sautreuil P, Maurisset M, Darmon L, Fode P, Lavaste F. A functional evaluation of prosthetic foot kinematics during lower-limb amputee gait. Prosthet Orthot Int 2006; 30:213-223

19. Dou P, Jia X, Suo $S$, Wang $R$, Zhang M. Pressure distribution at the stump/socket interface in transtibial amputees during walking on stairs, slope and non-flat road. Clinical Biomechanics 2006; 21: 1067-1073

20. Shuxian Z, Wanhua Z, Bingheng L. 3D reconstruction of the structure of a residual limb for customising the design of a prosthetic socket. Med Eng Phys 2005; 27:67-74 